\title{
Pattern recogaition, image processing and computer vision in fifth generation compater systems
}

\author{
D DUTTA MAJUMDER \\ Electronics \& Communication Sciences Unit, Indian Statistical Institute, \\ Calcutta 700035 , India
}

\begin{abstract}
It is well-known that one of the goals of research for the last two decades or so in pattern recognition and its sub-areas, such as processing, analysis and understanding of image, speech and natural language, and computer vision techniques etc., has always been to develop fundamental techniques for flexible interactive intelligent man-machine interfaces for computers. In this paper, the author tries to argue that for the evolution of fifth generation computer systems (FGCS) as defined by Japanese scientists, some of the things required are realisation and implementation of the advances in pattern recognition and its sub-areas, not only to achieve the man-machine interface with a natural mode of communication, but also for the realisation of the basic mechanisms of inference, association and learning, which are inherent both in pattern recognition and in the core functions of FGCS. The next generation computers will be knowledge-based systems, which form a subdomain of artificial intelligence (A1) techniques, and so Al provides the essential link between pattern recognition domains and different application systems. No attempt is made to discuss other essential conceptual building blocks, such as software engineering, computer architecture and very large scale integration technology unless these become very relevant in the discussions of concerned topics of the paper. A section on limitations of perception, learning and knowledge for computing machines is also included.
\end{abstract}

Keywords. Pattern recognition; artificial intelligence; image processing; computer vision; fifth-generation-computers; knowledge-based systems; man-machine-interface; speech recognition; language processing.

\section{Introduction}

During the joint session of the Eighth World Computer Congress and International Federation for Information Processing (IFIP) Congress 1980 in Tokyo (September 1980), a Japanese national project known as Pattern Information Processing Systems (PIPS) was presented. PIPS covered 12 main areas of research with more than 20 
application programmes divided into 4 main parts-(1) devices and materials, (2) information processing systems, (3) integrated system prototype and (4) pattern recognition systems - and involved around 10 thousand man-years of work. There were different reactions to this project among the commentators and scientists, though many felt that PIPS will eventually occupy a prominent place in the history of information technology. During the same IFIP congress some preliminary information about a nascent national computer project on fifth generation computer systems (FGCS) was also distributed (details in Moto-Oka et al 1981, pp. 1-85).

In 1979 the Japanese constituted a task force drawn from various universities and industrial and national research laboratories, which was charged with the task of formulating the image of the computers of the '90s. This task force reviewed a 10-year research project divided into three periods of 3,4 and 3 years that would lead to the development of fifth generation computer systems. The project started in 1982 and the programme is now being carried out by the Institution of New Generation Computer Technology (ICOT). In the last 3-4 years there have been several national, co-operative and corporate efforts in this field outside Japan, in the USA, UK, FRG, France, EEC, and also India, as a result of which a new framework of R \& D is emerging in Information Technology which will differ from past $\mathbf{R} \& \mathbf{D}$ environments, and papers on diverse aspects of FGCS research are being published. No attempt will be made here to present a complete review of the status of FGCS research.

From the information depicted in the first two paragraphs it should be clear that the project PIPS, the main motivation of which was to investigate the $R$ \& $D$ requirements in terms of devices, circuits and systems (hardware, software logic and mathematical algorithms) for Pattern Information Processing, is of crucial importance in the FGCS R \& D programme. Also, a certain amount of "human-like" intelligence or the capability to learn and gather knowledge from the continuous processing and handling of information patterns, needs to be incorporated into the next generation of computers.

If we could have an acceptable science of intelligence, or an information processing theory of intelligence in cognitive sciences, it could guide us in the development of the design technology of intelligent machines as well as explicate intelligent behaviour as it occurs in humans or other animals. since the development of such a general theory is still very much a distant goal, we should limit our attention to those principles that are relevant to the engineering goal of building intelligent machines. And, in my view, in this process we can contribute more to the development of the general theory of natural intelligence, as speech pattern recognition and computer vision experiments in the last two decades have contributed to speech and image understanding processes in living beings.

However, it can be stated that the next generation computer will be a general Information Technology System evolved from unification of several current state-ofthe-art concepts, where no individual subsystem need be identified as the next generation computer. Intelligent interfaces will make communication easy, whereby identification of a typical device becomes irrelevant, and knowledge-based systems can extend the range of services that computers can perform. At the intellectual level, some of the current disciplines will merge, and some new disciplines will emerge.

In this paper, an attempt will be made to highlight some salient features of FGCS research in relation to some of the selected topics, as mentioned in the title, and their relevance to objectives, architectures and applications. 


\section{Social and technical objectives of FGCS research}

It is well-known that computers were designed by mathematicians and engineers mainly to solve numerical problems and even in fourth generation computers with very large scale integration (VLSI) architecture there has not been any significant change in that respect. However, if we conduct a survey about the information generated as a result of the interaction between modern science and society, which needs to be processed for decision making purposes in different sectors of the society, we are bound to conclude that more than $80 \%$ of the information is non-numerical in nature, e.g., natural languages, speech sounds, printed characters, cursive scripts, photographic images, ECG, EEG, EMG etc. X-ray photographs and many other diverse non-numerical documentary pieces of information. Present day computers have not yet been able to demonstrate their processing power in these applied fields in any satisfactory way. Future computer systems would be expected to have the capability to obviate these difficulties and will be used to process these non-numerical data in different fields of applications, interfaced with the numerical processing capability of 4 th generation computers. A possible list of areas of application of FGCS including current ones (Dutta Majumder 1983) may be as follows:

1. Man-machine communication-(a) Automatic speech recognition, (b) Speaker identification and recognition, (c) OCR systems, (d) Cursive script recognition system, (e) Speech understanding system, (f) Image understanding, (g) Natural language processing.

2. Bio-medical applications - (a) ECG, EEG, EMG analyses, (b) Cytological, histological and other stereological applications, (c) X-ray analysis, (d) Diagnostics, (e)Mass screening of medical images such as chromosome slides for detection of various diseases, cancer smears, X-ray and ultrasound images and tomography, (f) Routine screening of plant samples.

3. Applications in physics - (a) High energy physics, (b) Bubble chamber and other forms of track analysis.

4. Crime and criminal detection - (a) Fingerprint, (b) Handwriting, (c) Speech sound and (d) Photographs.

5. Remote sensing and natural resources study and estimation - (a) Agriculture, (b) Hydrology, (c) Forestry, (d) Geology, (e) Environment, (f) Cloud pattern, (g) Urban quality, (h) Cartography, the automatic generation of hill-shaded maps, and the registration of satellite images with terrain maps, (i) Monitoring traffic along roads, docks, and at airfields, (j) Exploration of remote or hostile regions for fossil fuels and mineral ore deposits.

6. Stereological applications - (a) Metal processing, (b) Mineral processing, (c) Biology and (d) Mineral detection from microphotographs of ore sections.

7. Military applications - All the above six areas of applications plus (a) Detection of nuclear explosions, (b) Missile guidance and detection, (c) Radar and sonar signal detection, (d) Target identification, (e) Naval sub-marine detection, (f) Reconnaissance application, (g) Automatic navigation based on passive sensing, (h) Tracking moving objects, (i) Target acquisition and range finding.

8. Industrial applications - (a) Computer assisted design (CAD) and manufacture, (b) Computer graphic simulation in product testing, (c) Automatic inspection in factories, (d) Non-destructive testing, (e) Object acquisition by robot arms, for example by "pin 
picking", (f) Automatic guidance of seam welders and cutting tools, (g) Very large scale integrated related processes, such as lead bonding, chip alignment, and packaging, (h) Monitoring, filtering and thereby containing the flood of data from oil drill sites or from seismographs, (i) Providing visual feedback for automatic assembly and repair, (j) Inspection of printed circuit boards for spurs, shorts, and bad connections, (k) Checking the results of casting processes for impurities and fractures.

9. Robotics computer vision and artificial intelligence - (a) Intelligent sensor technology, (b) Natural language processing, (c) All computer vision applications, (d) Object acquisition and placement by robots, (e) Designing expert systems for specific applications that require non-numerical information handling.

10. Management applications - (a) Management information systems that have a communication channel considerably wider tkan current systems that are addressed by typing or printing, (b) Document reading and other office automation systems.

From a cursory glance of the above list one can summarise that the role of FGCS is to enhance productivity in non-standard operations in the tertiary industries, overcome constraints on resources and energy consumption, realise mass level health-care, education and other support systems, and step towards a transition to a world society.

From this incomplete list of application areas we should also conclude that FGCS research should be aimed at two major objectives - the first is the social objective, namely, to reduce or eliminate the alienation between man and machines, and that machines should be made as inexpensive as possible, and the second is the technological objective of overcoming the deficiencies in the processing of huge amounts of non-numerical information. The Japanese task force suggested a systems approach known as the knowledge information processing system (KIPS) which will support a high logic level and at the same time is friendly with and familiar to human beings. KIPS will have knowledge bases and will be able to infer from stored knowledge and solve problems, and take decisions in a way similar to humans. Such knowledgebased systems will evolve out of present day machines, which are designed around numerical computer systems. But these new machines will have the ability to access the meaning of information and understand problems, described in human language, and solve them such that human beings are aided in their different socio-economic tasks at a higher level of intelligence.

\section{Evolution to the next generation computing system}

For this evolution to the next generation some of the things required are practical implementation of the advances in pattern recognition, image analysis, computer vision and artificial intelligence, not only to realise the man-machine interface with a natural mode of communication, but also for the realisation of the basic mechanism for inference, association and learning, which are inherent in pattern recognition, image analysis, computer vision and artificial intelligence research, and the methodology to form the core function of the fifth generation computer.

The next important point is the realisation of enhanced software productivity and the application of artificial intelligence (AI) techniques to utilise the above functions along with retrieval and management of knowledge-bases in hardware and software.

It is needless to state that in order to equip these FGCS of tomorrow with human type senses and logical processes, larger and faster chips than the current vLSI must be 
fabricated, and chip designers are therefore looking forward to the production of superchips by ultra large scale integration (ULSI).

It is estimated that it will be possible to place approximately 10 million transistors on a single IC chip. At present the sizes of the chips vary from 5 and $7 \mathrm{~mm}$ on a side for most complex functions. By 1990 the size may be increased to $25 \mathrm{~cm}$ on a side, and the size of the individual features used for the circuits on the chip will be approximately 1 micrometre (one millionth of a metre) which means approximately 100 million rectangular shapes on the chip surface. Previously these shapes have been manually specified for the designs. For a reasonably sized design team it is impossible to carry out the job in a way that can be expected to lead reliably to circuits that satisfy the desired function. Though basic fabrication technology is capable of implementing these shape features, to provide methods such that a designer can quickly, correctly and economically convert a high level functional specification into an accurate representation of shapes that will lead to properly functioning circuits is a challenge which can be met by designing an "Intelligent ULSI-CAD" system and associated inspection mechanisms incorporating the latest results of shape analysis, pattern recognition, computer vision and robotics.

Apart from that, as we have little guidance as to how such a high-level description should be formally specified, substantial experimentation with the variety of formal languages which are known as hardware design languages (HDL) is needed before any consensus can be obtained about the best means of expression.

It should be understood that interplay between performance strategy, functional specification, architecture and choice of technology (CMOS, NMOS or bipolar current mode logic-such as ECL) are of overriding importance. There are even more exotic technologies, such as the use of superconducting Josephson junctions (Allen 1983), or the use of gallium arsenide instead of silicon as a semiconductor. It can be safely expected that in FGCS research all these are being explored, though practical systems will be built using silicon as a semiconductor substrate, in either NMOS or CMOS or some hybrid technology that combines the virtues of both.

\section{Overview of FGCS and intelligent interface system}

The main functions of fifth generation machines can be broadly classified under three headings: (1) problem-solving and inference-making functions, (2) knowledge based management functions and (3) intelligent man-machine interface functions.

These functions will have to be realised by making individual software and hardware subsystems corresponding to the general FGCS framework. A conceptual framework of the system (Moto-oka et al 1981) is shown in figure 1. The descriptions of the blocks in the diagram are to some extent self explanatory. In this diagram the upper half of the modelling (software) system circle corresponds to problem-solving and inference functions, the lower half to the knowledge-based machine system (KBMS) functions.

The portion that overlaps the human system circle corresponds to the intelligent interface function. From this diagram it is clear that the intelligent interface function relies heavily on the two former groups of functions.

In my view high speed computer communication Dutta Majumder (1984) and local area networking will also constitute an important infrastructure in the final FGCS usage as shown in the modified version (figure 2 ) 


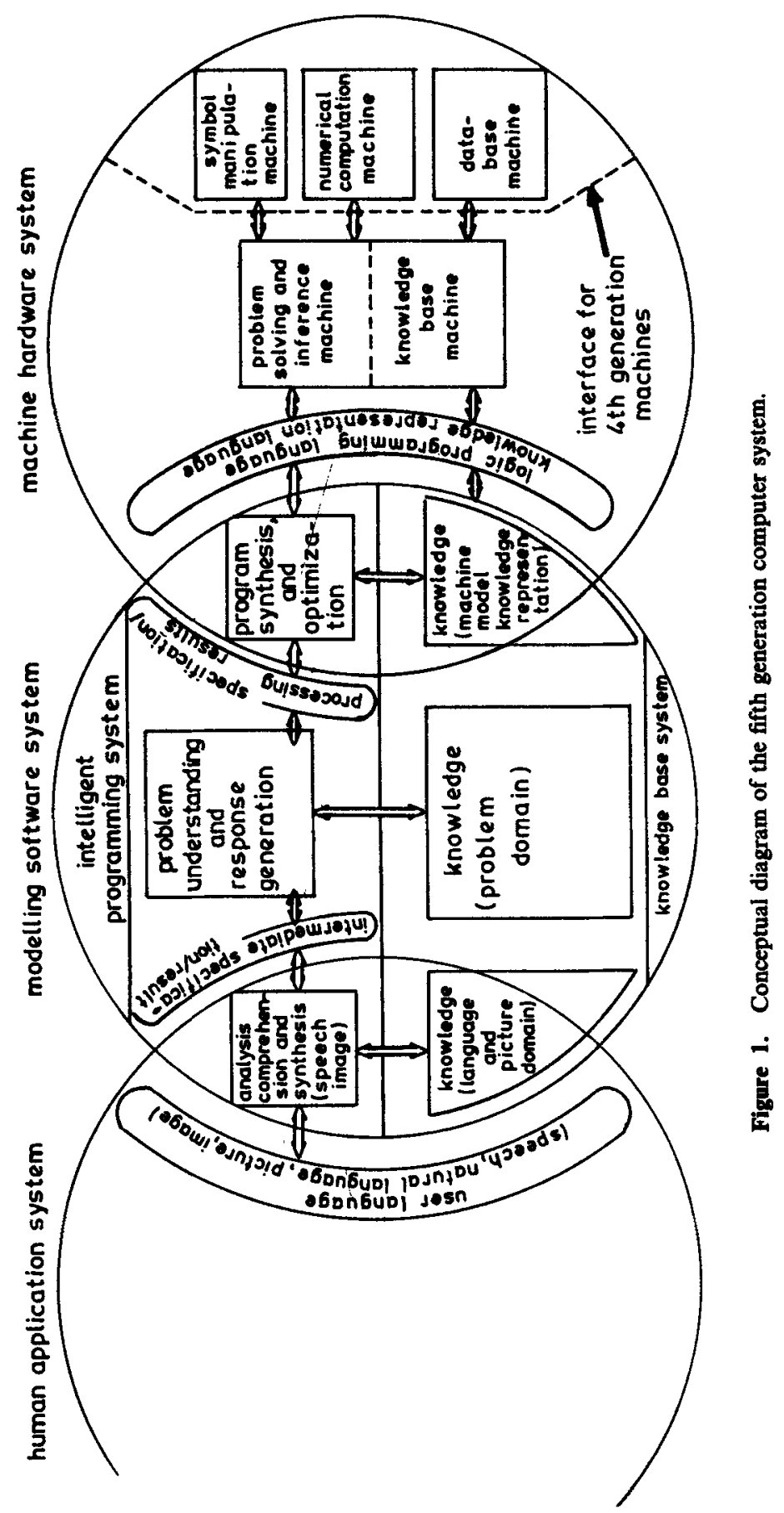




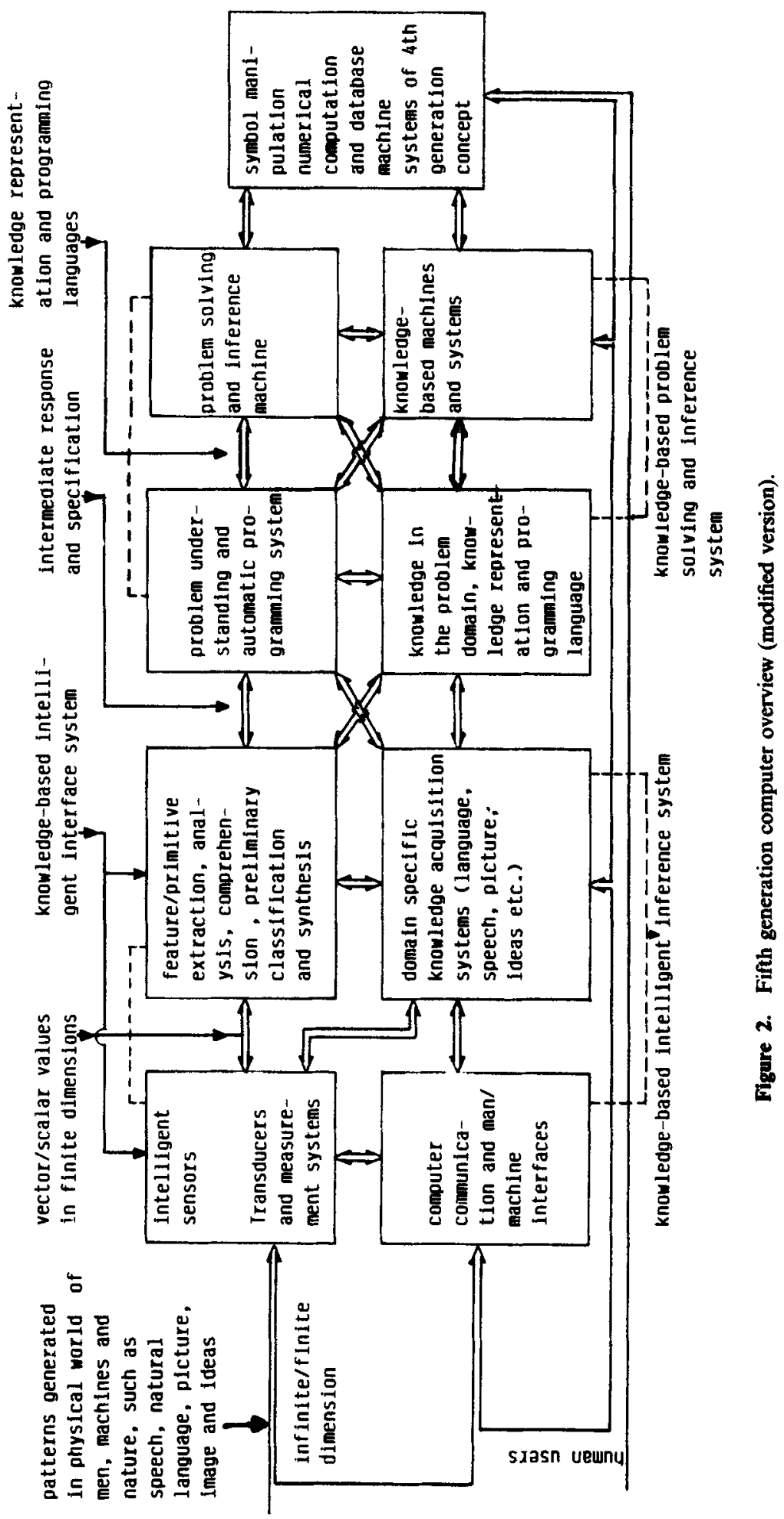


A problem, as presented by the application system, through some end-users language that can use voices, figures and images etc. is analysed, recognized/understood by using the knowledge about the language and the images/pictures. This is then translated into intermediate specifications, which are given to the programming system. Here an attempt is made to understand the problem, using the knowledge about the problem domain, and as a result processing specifications are formulated. The specifications are transformed into a program and optimized through referencing the knowledge about the machine system and the knowledge-representation. The program, written in some logic programming language, is then processed by the problem-solving and inference mechanisms and the knowledge-base machines. The numerical computations, symbolic manipulations and database machines in figure 2 are coprocessors of the problem-solving and inference, as well as the database machine.

Though all these three or four functions mentioned above are integrally related to each other, the defined plan for developing an intelligent interface comprises - (a) pattern recognition, image processing and understanding, (b) natural language processing and (c) automatic speech recognition and understanding (Dutta Majumder \& Dutta 1968; Fu 1968, 1982; Liberman 1970; Lea 1980; Haton 1982; Underwood 1983; Dutta Majumder \& Pal 1985).Actually in the FGCS scheme the intelligent manmachine interface system constitutes a front-end processor for the input/output using spoken and written natural languages, pictures and images, as shown in figures 1 and 2 giving the basic configuration and the conceptual structure of FGCS. In the theoretical framework FGCS should imply a unified approach of cybernetic and general systems theory (Dutta Majumder 1979).

The FGCS aim of developing systems that are highly user-friendly suggest that current high level computer languages are inadequate for many purposes. A corollary to this interpretation is that natural languages (English, Japanese, Hindi, French, Bengali etc.) will become the ultimate programming languages assuming that a sufficiently intelligent man-machine interface can be designed. Existing natural language systems are still less flexible than normal English and make more demands on users. These systems work on a limited vocabulary where programs are fed into the system via a key-board. One purpose of FGCS research will be to overcome the limitations of existing natural language systems because the demand for oral communications in FGCS requires speech recognition, speaker identification and speech understanding systems.

In order to provide flexible interactive intelligent man-machine interfaces in the final FGCS the plan for research will have to be motivated to develop fundamental techniques in all the three categories of pattern recognition research, namely, natural language processing, speech processing, and graph and image processing. However, in the research and development stage, state-of-the-art terminals will have to be used in all the FGCS projects because an intelligent man-machine interface system will itself be a kind of KBMS composed of a front-end processor of various input/output forms, flexible KBMS and problem solving/inference systems. However, in the FGCS context we use the term "intelligent interface system" to denote the front-end processor for input/output in forms such as natural languages, both spoken and written, pictures and images (computer vision). 


\section{Perception, learning and limitations of knowledge for machines}

Again, if we look back at the history of modern computer science and information technology, we can recognise two major approaches: that of the so called 'hard' school and the 'soft' school. Members of the first group are concerned with building a strong theoretical component to their work based on pure mathematics. Members of the second group consider that the strong theoretical component is not only unnecessary but positively harmful. The first group on the other hand consider the work of the second school as just mundane application. Practical realisation, however, usually accrues from theoretical and experimental co-ordination of the findings of both schools.

Innovations often come from the reassessment of old ideas from both schools. The development of succeeding generations of computers is marked by a fresh look at current activities and this encourages extensions of the techniques employed. Sometimes these new ideas occur well before the technology can support them or the mathematical tools and techniques are well developed for the purpose. Consequently these ideas remain in the backwaters of mainstream science waiting to be re-discovered. Notable examples are the ideas of Charles Babbage and of Turing.

The FGCS specifications about Inference machines and knowledge-based systems, on the surface appear to be influenced by the "hard" school. The important results of pattern recognition (PR) and AI in the last decade that interests designers has been to show that a higher level of problem specification can be achieved by engineering 'knowledge' and pattern directed inferences and it is this principle that should underlie new design objectives.

In the last four decades since the advent of digital computers there has been constant effort to expand the domain of computer applications. Pattern recognition is an area of activity to process the huge amount of non-numerical information generated as a result of the interaction between science and society. Computer scientists are interested in designing machines that can speak and write, and have understanding like human beings. This area of activity gave rise to what is now known as artificial intelligence. Both these motives are inherent in the area we sometimes call machine learning (ML) or machine perception (MP).

At present the ability of machines to perceive their environment is very limited. A variety of transducers are available for converting sound, light, temperature, pressure etc., to electrical signals. When the environment is carefully controlled, the perceptual problems become trivial. But as we move beyond having a computer read magnetic tapes to having it read hand-printed characters or analyze biomedical photographs, we move from problems of sensing the data to problems of interpreting and understanding them.

The apparent ease with which vertebrates and even insects perform perceptual tasks is both encouraging and frustrating. Psycho-physio-physical studies have given us many interesting facts, but not enough understanding to duplicate their performance with a computer. We are all experts at perception but none of us know much about it. Because there is no general theory of perception, we have had to start with modest problems. Many of these involve pattern classification - the assignment of a physical object, event or idea to one of several prespecified categories. Extensive study of classification problems led to some mathematical models (Dutta Majumder \& Dutta 1968; Fu 1968, 1982; Lea 1980; Dutta Majumder \& Pal 1985) that provide the 
theoretical bases for classified designs. Of course, in any specific application one ultimately must come to grips with the special characteristics of the problem at hand. A general mathematical theory of pattern recognition and machine learning is yet to be formulated.

\subsection{Limitations of knowledge for machines}

Without entering into the brains, machines and mathematics (Arbib 1964) controversies, it can be safely argued that these controversies relate to our logical mind, whereas we have other inspirations and experiences that give us a clue to deeper levels of consciousness and intelligence. Most of the neurophysiological theories and mathematical models so far are based on grossly simplified views of the brain and the central nervous system. There is a variety of properties - memory, computation, communication, control, learning, purposiveness, reliability despite component malfunction - which it seems difficult to attribute to mere mechanisms. The mind and intelligence we ordinarily use is limited to reception of sensory data from the outer physical world (and usually not the inner mental world) which we use to assemble, observe, control, regulate and communicate, for the purpose of learning, organising, planning and calculating analogous to a computer. Published literature on FGCS research from Japan and elsewhere more or less concerns this logical mind which is attempted to be made computer (IBM)-compatible!

In his famous incompleteness theorem Godel (1931) has shown the limitations of the logical process. According to Nagel and Newman (1958) the axiomatic method, which lies at the foundations of our modern theory of logic programming and probability has certain inherent limitations. They proved that it is impossible to establish the internal logical consistency of a very large class of deductive systems. Sir Arthur Eddington $(1939$, p. 148) terms the logical mind as "the group structure of a set of sensations in a consciousness?" Late Nobel Laureate Professor Dennis Gabor's (1960) compromise formulation is "I have a consciousness, which receives sensory data from an outer, real, physical world, and images, concepts and urges from my unconscious mind". This partition of mental structure to conscious and unconscious mind does not seem to me to be a realistic concept. It is more likely that there are different levels of consciousness which are interactive in nature from unconscious, extra conscious, super conscious and other noncognitive levels of awareness to ordinary consciousness which performs dayto-day information processing, and motivates psychodynamic activities.

Without attempting to put forward any coherent theory of intelligence, it can be safely argued that the nature of intelligent messages (Fatmi 1984) in different types of flashes of inspiration and other usual experiences is entirely different from the artificial intelligence of the FGCS Logic Programs talked about in the literature. It should be understood that all this is at a far lower level than that exhibited by a human being, and that many differences between man and machine are not only immutably qualitative but enormously quantitative. To even partially bridge this gap some kind of theoretical breakthrough will be needed.

\section{Computer vision and image/picture processing and understanding}

Computer vision (CV) and image/picture processing and understanding (IU) constitutes one of the most important functions in intelligent man-machine interfaces. In order to 
reduce the alienation between man and machine one of the most innate capabilities of humans, to see and understand extremely complex scenes at a glance, should be incorporated into machines. Second, the system should be provided with an environment in which the user can handle pictorial and image data as a knowledge source.

In the present state-of-the-art in this field, even the best computer vision systems are somewhat primitive in their capabilities. Under strictly constrained conditions they can recognise and locate objects on a conveyor belt. The development of a general purpose computer vision system that can approach the abilities of the human eye and brain is remote at present, despite recent progress in understanding the nature of vision.

Factors contributing to difficulties in the vision system may be grouped as (a) insufficient information in the image, (b) the reflectance properties of the objects, (c) the nature of illumination, (d) the viewing angle available, (e) securing adequate knowledge for fitting together of objects for interpretation, and (f) the large computation and memory space required.

From the list of current and potential applications indicated in $\$ 2$ of this paper, it can be observed that the areas of image processing, IU and CV probably provide the most stimulating research area for the application of the rich theories developed in the fields of statistical, syntactic and fuzzy set theoretic approaches of pattern recognition, and the concepts and ideas of artificial intelligence. The characteristic feature of IU is the construction of rich descriptions from an image, and hence, there exist significant overlaps between IU, CV and image processing, especially in the early processing operations of edge detection and region finding. Computer graphics is also particularly involved in the area, primarily with the display of visual information. The results of all these developments will have to be integrated and incorporated in the intelligent interface system of FGCS. The intellectual climate for progress and the theoretical base for IU and allied fields has improved with the work conducted under the US Defence Advanced Research Project Agency's (DARPA) IU program.

Apart from that, there exist good text books (Fukanaga 1972; Duda \& Hart 1973; Newman \& Sproull 1973; Prat 1978; Faux \& Batt 1979) and review and research papers encompassing this whole area of activity (Falk 1972; Haralick 1973; Rosenfeld \& Kak 1976; Birk et al 1978, 1981; Bribiesca \& Guzman 1980; Dutta Majumder \& Chaudhuri 1980; Vamos \& Bathor 1980, pp. 1091 -93; Parui et al 1980; Barhard 1981; Levine \& Shaheen 1981; Parui \& Dutta Majumder 1982, 1983; Anon 1984; Chanda et al 1984; Chanda \& Dutta Majumder 1985; Dutta Majumder 1985). This is not, however, an exhaustive list of references.

Figure 3 indicates the pattern of representations and modules describing the computational approaches in different levels of image understanding, which can also be viewed as a flow diagram. The diagram suggests that the flow of information and the construction of representations are entirely sequential, proceeding from the lowest level operations on the image to more semantic higher level operations. It may be true that our perceptual process is not rigidly sequential, and context along with pragmatics plays an important role in our perceptual process, which is also true of our speech perception mechanism.

In the next section, we discuss the status of current and potential applications of $\mathrm{CV}$ techniques in some industrial applications, particularly robotics and industrial inspection systems. 


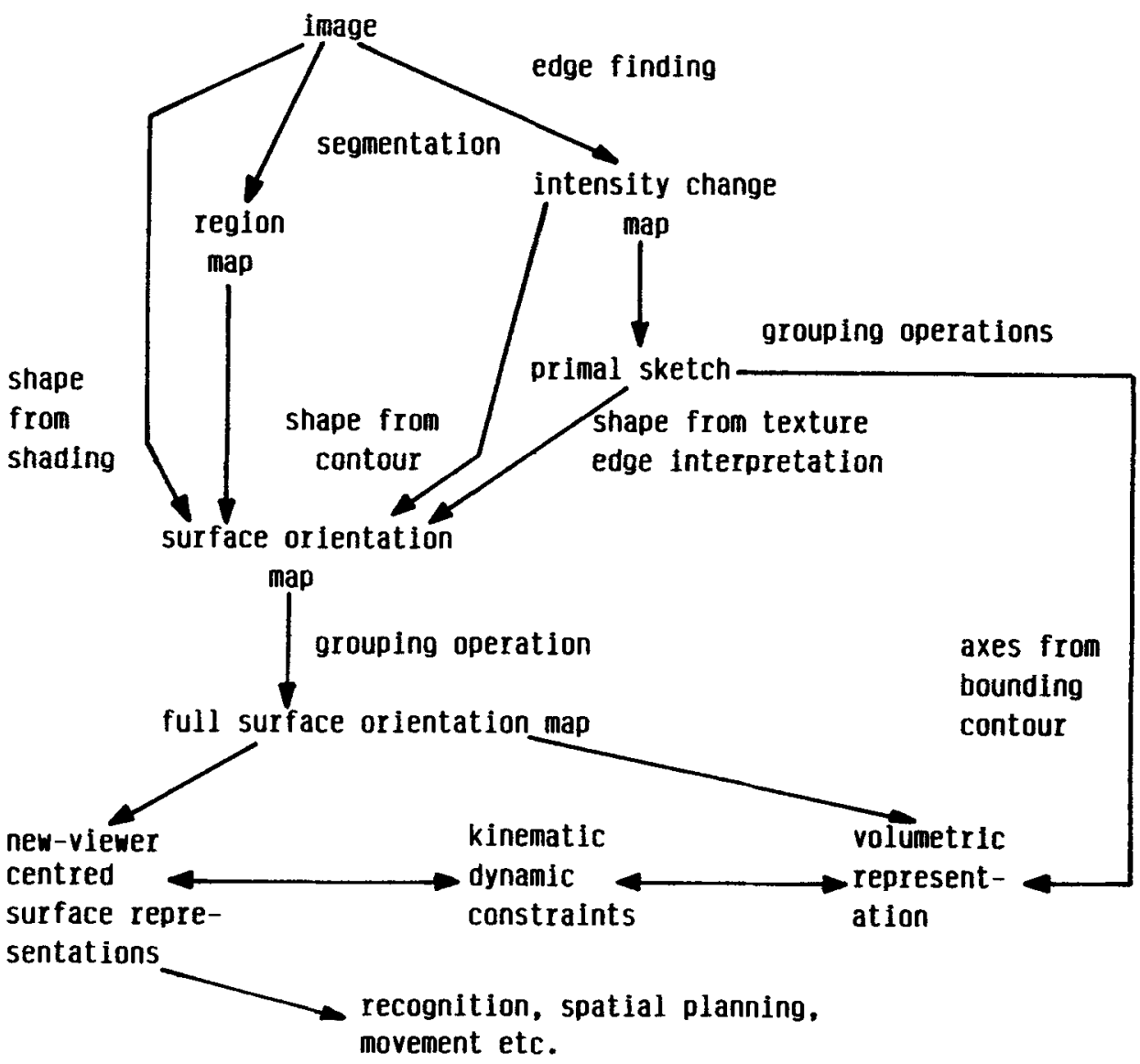

Figure 3. Computational approaches to image understanding; some of the representations and modules discussed in the paper.

\subsection{Computer vision in FGCS for industries}

Computer vision techniques involve visual sensing, imaging devices, imaging methods, image processing techniques, such as segmentation, edge detection, thresholding, region growing, image description, and ultimately image recognition and understanding the application of pattern recognition techniques. Pattern recognition and understanding also involve feature, texture, hole, and shape detection and extraction, and other highly specialized mathematical and engineering techniques.

All the above terms involve complex computational algorithms that need not be understood by most people. But it should be understood that some kind of communication must be established between the user and the computer-based robotic system so that the user can direct the manipulator to perform a given desired task. There are several ways that are being studied to communicate with robots, such as (a) discrete word recognition, (b) teaching the robot by leading it through spaces while using a reach box, (c) programming in high level languages, (d) natural language interactive programming. This is more or less the subject of robot control languages, which depends largely on artificial intelligence techniques and pattern recognition methods. 
Artificial intelligence is the field that aims to understand how computers can be made to exhibit intelligence in different aspects of thinking, reasoning, perception or action in some limited sense. A key contribution of $\mathrm{AI}$ is the observation that knowledge should be represented explicitly and not be heavily encoded, such as numerically, in ways that suppress structure and constraint. AI has developed a set of techniques such as semantic networks, frames and production rules, that are symbolic, highly flexible encoding of knowledge but yet can be efficiently processed.

In robotics, perception systems produce geometric models of real world situations, and reasoning systems deliberate on those models while action systems interpret them. Computer aided design (CAD) and computer aided inspection systems have been concerned with highly restricted uses of geometric information.

In so far as robotics is the intelligent connection of perception to action, $\mathrm{AI}$ is the challenge for robotics. On the other hand, one can be assured that robotics challenges AI by forcing it to deal with real objects in the real world, though techniques and representations developed for purely cognitive problems often do not extend to meet the challenge.

AI has also uncovered techniques for using knowledge effectively. One problem is that the knowledge needed in any particular case cannot be predicted in advance. Programs have to respond flexibly to a non-deterministic world. Among the techniques offered by AI are search, structure matching, constraint propagation, and dependencydirected reasoning. Of course, different techniques developed by AI research need to be modified/extended for application in real world robotics problems.

All techniques are exploited to develop better robots or intelligent machines that will solve problems (action planning) and will perceive things as we humans do. The subject of machine intelligence and robot planning is intimately related to the fields of artificial intelligence, automatic control, pattern recognition and computer vision. With the realization of FGCS technology a new generation of intelligent robotics is emerging. To obtain a system that not only speeds up the planning process but also increases problem-solving capability for handling complex and varied tasks in the general world environment is a challenge for pattern recognition scientists.

\subsection{Computer vision in industrial inspection systems}

The recent widespread interest in computer vision techniques, robotics and computer controlled inspection and monitoring systems originated in USA and UK because of the industry's fundamental problem of stagnation. From 1947 to 1965 us productivity increased at an average annual rate of $3.4 \%$, which decreased to $2.3 \%$ in the following decade. It then dropped to below $1 \%$ and continued so till 1980 . Japan's productivity growth has been climbing at an average annual rate of $7.3 \%$ for all those years. It is well-known that there are different ways of influencing manufacturing productivity and quality such as financial, fiscal, social and technological. Therefore, our emphasis here is on the technological aspects, particularly industrial inspection systems some of which are applicable to selected sectors of industries in developing countries like India.

For an industrial inspection system to be viable, it must in general achieve the same or lower costs compared to those incurred by manual methods with better quality control and must have a reasonable inspection rate, as well as being reliable and maintainable. 
In the system under discussion the object to be inspected is illuminated and then imaged by a camera. The processor may include a subsystem for data reduction, since the data rate may be too high or the required memory may be too large. In addition to the implementation of the inspection procedure, the processor may perform other control and timing tasks. It may provide statistics on the number of parts inspected, on the classification of defects, and on the status of the system. There are three broad techniques of automatic computer vision inspection methods.

The first technique involves storage of a set of invariant features extracted from the defect-free image of the defect free object. The same set of features are extracted from the object under inspection and are compared to the former set to determine whether the object is defect free or not.

The second procedure involves a kind of windowing technique. If the part of the image inside the current window area violates some generic properties of the product under inspection, the product is defective. The generic properties are inferred from the defect free product.

In the third technique the image of the object being considered is compared to the defect free image of the object pixel by pixel. If the gray-level difference of any two pixels from the two images exceeds a threshold, then a defect exists at that pixel. This method is widely used in industrial inspection systems because it is simple, fast and reliable.

The third method of pixel-by-pixel comparison requires the storage of complete defect free images, and requires perfect alignment of two images, and is not flexible enough for many inspection tasks.

The most powerful method, however, is the first one mentioned above in principle but which requires extensive computational capability.

The second technique is successful only when the inspection criteria can be transformed into a set of rules that can be applied uniformly throughout the image.

The computer vision process may be divided into five principal areas - sensing, segmentation, description, recognition and interpretation. It should not be implied that human vision and reasoning can be so neatly subdivided or that these process are performed independently of each other. For example recognition and interpretation are highly interrelated functions in human beings, but this interrelationship is not understood to the point that they can be analytically modelled. The above subdivisions of functions should be looked at for limited practical implementation of state-of-theart computer vision systems.

In some sense, we may consider computer vision techniques as being arbitrarily divided into three basic levels of processing - low, medium and high-level vision. Lowlevel vision techniques attempt to extract "primitive" information from a scene ranging from modelling the characteristics of incident and reflected light properties of a body to the detection of edge segments in a scene. Medium-level vision refers to procedures that use the results from low-level vision to produce structures that somehow carry more meaning than that carried by elements extracted from a low-level vision process, which include edge linking, segmentation, description and recognition of individual objects. High-level computer vision may be viewed as the process that attempts to emulate cognition, encompassing a considerably broader spectrum of processing functions.

So far, industrial applications of the computer vision technique have been mostly at the low and medium level of processing, and a discussion on higher-level modelling is somewhat futuristic from the point of industrial applications. 
Computer vision is a dynamic field which has encompassed unified applications of pattern recognition, image processing and artificial intelligence techniques extensively.

\subsection{Status of 3-D shape determination}

Though successful programs for determining 3-D shapes from arbitrary 2-D images is yet to be developed, sufficient progress has been made such that systematic analysis of images from restricted types of scenes can be made. Modules have been developed that use shading, texture, contours, shadows, stereo-optical disparity, and other image features for the purpose, and apply different levels of vision discussed in the earlier section.

Several approaches have so far been taken to match models and images and the basic approach to the problem of object recognition and scene analysis is called model-based vision. There is a similarity with speech recognition where we try to match the models (words) with incoming speech signals. But the difficulty is that an object looks different from different angles, and so the program must be able to manipulate the model in three dimensions. In the next section, we present very briefly the status of spatial scene analysis in the context of the organisation of an aerial image analysis system, which is very important from the point of designing intelligent interfaces in FGCS for specific applications for countries like India.

\subsection{Spatial analysis and reasoning system}

In order to interpret a pictorial entity, generally speaking, we may need two types of information, namely, its intrinsic properties such as size, shape colour etc. and its relations to other entities. Specially for recognition of man-made objects in a scene, such as in urban, airport or dock areas, our primary interest is the representation of geometrical relations among objects and their utilization for image interpretation. Moreover, although shape can often be regarded as an intrinsic object property, a complex shape is often described structurally in terms of geometric relations among its components. Thus shape recognition often requires spatial analysis.

There should be three levels of representation and control in a shape analysis and reasoning system. First, a high level expert (HLE) that utilizes a symbolic hierarchical model for the possible spatial organization of objects in the image to build partial, local interpretations of the image and to determine where to further analyze the image and what analysis to perform; second, a model selection expert (MSE) that determines, on the basis of contextual information provided by the HLE, the most promising appearance descriptions to use in searching for objects and structures in the image; and third, a low level vision expert (LLVE), that finds pictorial entitities that satisfy these appearance descriptions by selecting image processing methods to find the appropriate entities. The HLE is based on an "evidence accumulation" process for performing spatial reasoning.

It is also generally true that image understanding systems should incorporate both bottom-up and top-down analyses, and functional representation of geometric relations such as spatial relations (SP), part-whole (PW) relations, a-kind-of (AKO) relations etc. and hypothetical formation rules should be designed as such.

Figure 4 shows the organization of such an image understanding system. The following steps are iterated by the HLE. (1) Each instance of an object generates hypotheses about related objects using functions stored in the object model (frame). 


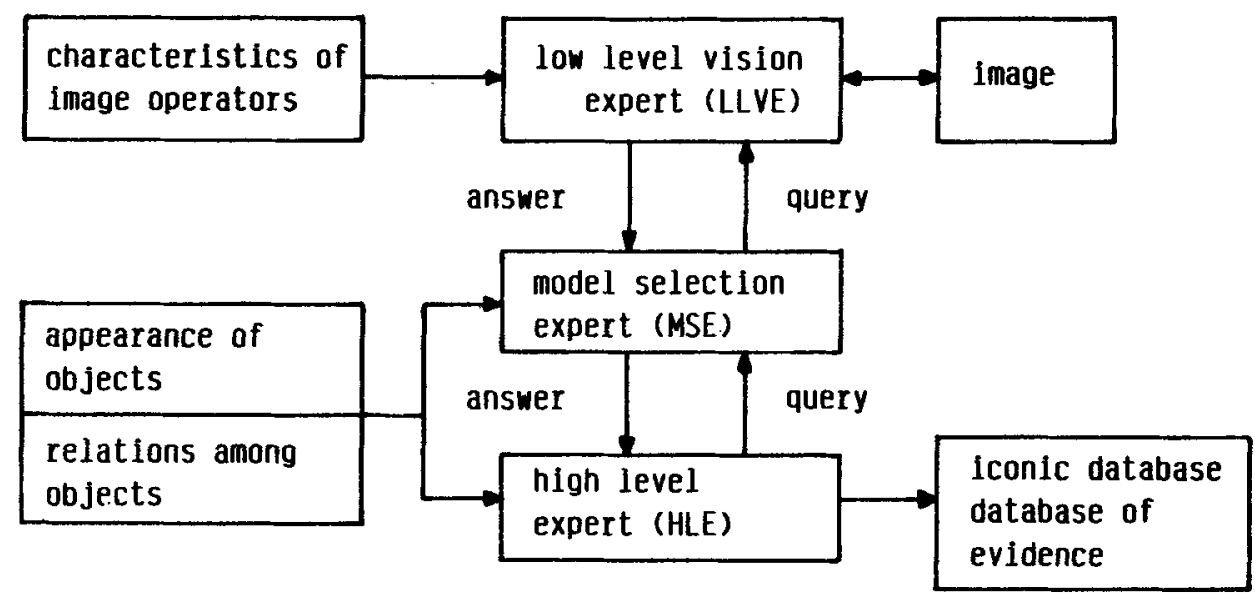

Figure 4. A knowledge-based image understanding system with three expert levels for combining evidence.

(2) All pieces of evidence, both instances and hypotheses, are stored in a common database (called iconic database) and represented using an iconic data structure which associates highly structured symbolic descriptions of instances and hypotheses with regions in a 2-dimensional array. (3) 'Situations' consisting of 'consistent' pieces of 'evidence' are established by their combination. (4) The most reliable situation is selected for focus of attention. (5) The selected situation is 'resolved' resulting either in verification of predictions on the basis of previously detected/constructed image structures or in topdown image processing to detect missing objects. The two additional processes are (7) 'Instantiation' of objects at the very beginning of interpretation is performed by the MSE which searches for object models that have simple appearances, and directs the LLVE to detect pictorial entities which satisfy the appearances. The 'instances' thus constructed are seeds for reasoning by HLE. (8) The HLE maintains all possible interpretations throughout the search process untill no further changes are made in the iconic database. A final interpretation then selects the maximal consistent interpretation.

From what has been stated in the above sections, it is clear that the general problem of recognizing objects in a scene and describing their relations in three dimensions is far from solved. Also there must be very significant increase in the speed of vision processors.

The mobile cart developed at Stanford University navigating at a speed of 3 to 5 metres per hour while analyzing one image of its surroundings for each 1-metre larch needed a 1 million instruction per second computer. To guide a similar cart at a walking speed of a metre per second will therefore require from $10^{9}$ to $10^{10}$ instructions per second. Of course there are many social and day-to-day applications which do not require such high speed processing.

\section{Conclusions}

The purpose of this paper has been to outline the role and impact of pattern information processing research such as inference, estimation, and recognition 
procedures in statistical, syntactic and fuzzy set theoretic approaches in general pattern recognition problems of speech, natural languages, pictures, images etc. in future generations of computer systems research. We have attempted to show that the developments in PR and AI in the last two decades are not only crucial for intelligent interfacing machines but also for the realisation of core functions of KBMS and inference engines. It should also be understood that with the typical next generation system no single item of technology should be identified as the next generation computer. But the most important subdomains are AI-based KBMS, language understanding and speech and picture recognition. Language understanding can be useful as interpreters for other programs or for translation. Speech and picture recognition will not only speed up the input to the computer but will revolutionize the use of autonomous devices - robots that plan their actions in response to their environment, or in industrial manufacturing systems. Another point to be noted is that the right kind of architecture to achieve these goals is still an open question which we have not dealt with at all. We have also tried to indicate the limitations of different concepts and technologies and the need for breakthroughs in some fields.

This work was funded by the Knowledge-Based Computer Systems Project of the Department of Electronics, Government of India.

\section{References}

Ahmed I, Fung K T 1981 Computer science (Potomac, Md: Computer Science Press)

Allen J 1983VLSI overall system design, FGCS state-of-the-art report, Pergamon Infotech Rep.

Anon 1984 Spatial reasoning Tech. Rep. CAR-TR-28, University of Maryland, USA

Arbib M A 1964 Brains, machines and mathematics (New York: McGraw Hill)

Barnard S T 1981 Proc. IEEE 69: 572-595

Birk J, Kelley R, Martins H 1981 IEEE Trans. Syst., Man Cybern. 11: 151-160

Birk J, Kelley R, Wilson L 1978 Proc. 8th Int. Symp. on Ind. Robots, Stuttgart, West Germany (New York: Robot Inst. Am.) pp. 724-733

Bribiesca E, Guzman A 1980 Pattern Recognition 12: 101-112

Chanda B, Chaudhury B B, Dutta Majumder D 1984 IEEE Trans. Syst., Man Cybern. 14: 515-518

Chanda B, Dutta Majumder D 1985a Inst. J. Syst. Sci. 16: 71-80

Chanda B, Dutta Majumder D 1985b Pattern Recognition Lett. 3: 243-251

Duda R O, Hart P E 1973 Pattern classification and scene analysis (New York: John Wiley \& Sons)

Dutta Majumder D 1979 Kybernetik 8: 7-15

Dutta Majumder D 1983 J. Inst. Electron. Telecommun. Eng. 29: 429-449

Dutta Majumder D (ed.) 1984 Pattern recognition and digital techniques (Calcutta: Indian Stat. Inst.) pp. 499-529

Dutta Majumder D 1985 Pattern recognition and artificial intelligence techniques in intelligent robotic systems, Proc. First National Convention of Prod. Eng. Div of Inst. of Engineers, Calcutta, August

Dutta Majumder D, Chaudhuri B B 1980 Int. J. Syst. Sci. 11: 1433-1445

Dutta Majumder D, Datta K 1968 Indian J. Phys. 42: 425-443

Dutta Majumder D, Pal S K 1985 Fuzzy mathematical approach to pattern recognition (New Delhi: Wiley Eastern)

Eddington A 1939 The philosophy of physical sciences (Cambridge: University Press)

Falk G 1972 Artif. Intell. 3: 101-144

Fatmi H 1984 A theory of processing intelligent messages (London: University Press)

Faux I D, Batt M J 1979 Computational geometry for design and manufacture (Chichester: Ellis Horwood)

Fu K S 1968 Sequential methods in pattern recognition and machine learning (New York: Academic Press)

Fu K S 1982 Syntactic pattern recognition and applications (Englewood Cliffs, NJ: Prentice Hail) 
Fukunaga K 1972 Introduction to statistical pattern recognition (New York: Academic Press)

Gabor D, Wilby W P L, Woodcock R 1961 Proc. Inst. Elec. Eng. B108: 422-438

Godel $\mathrm{K} 1931$ On formally decidable propositions of principia mathematica and related systems (Transl.). B Meltzer (New York: Basic Books Inc)

Goldstein H H 1972 The computer from Pascal to Von Nevomann (Princeton: University Press)

Haralick R M, Shanmugan K, Dinstein I 1973 IEEE Trans. Syst., Man Cybern. 3: 610-621

Haton J P 1982a Proc. 6th Int. Conf. Pattern Recognition, Munich, October (Washington, DC: IEEE Computer Society)

Haton J P (ed.) 1982b Automatic speech analysis and recognition (Dordrecht: D Reidel)

Lea W A 1980 Trends in speech recognition (Englewood Cliffs, NJ: Prentice Hall)

Levine M D, Shaheen S I 1981 IEEE Trans. Pattern Anal. Mach. Intell. 3: 540-556

Libermann A M 1970 Cognit. Psychol. 1: $301-323$

Moto-Oka T, Tanaka H, Hirata K, Maruyama T 1981 Proc. Int. Conf. on FGCS, Tokyo, October (Amsterdam: North Holland)

Nagel E, Newman J R 1958 Godel's proof (New York: University Press)

Newman W M, Sproull R F 1973 Principles of interactive computer graphics (New York: McGraw Hill)

Parui S K, Chaudhuri B B, Dutta Majumder D 1980 J. Inst. Electron. Telecommun. Eng. 26: 21 -28

Parui S K, Dutta Majumder D 1982a Pattern Recognition Lett. 1: 129-134

Parui S K, Dutta Majumder D 1982 b How to quantify shape distance for 2-D regions, Proc. 7 th Int. Conf. on Pattern Recognition, Montreal, pp. 72-74

Parui S K, Dutta Majumder D 1983a pattern Recognition 1: 129-134

Parui S K, Dutta Majumder D 1983b Pattern Recognition 16: 63-67

Prat W K 1978 Digital image processing (New York: Wiley Interscience)

Rosenfeld A, Kak A C 1976 Digital picture processing (New York: Academic Press)

Turing A M 1937 Proc. Lond. Math. Soc., Second Ser. 42: 230-265

Underwood M J 1983 Intelligent user interfaces, Pergamon Infotech Repott.

Vamos T, Bathor M 1980 Proc. 5th Int. Joint Conf. Pattern Recognition(Los Alamitos, CA: IEEE Computer Society) 